\title{
Numerical Solution of Seventh-Order Boundary Value Problems by a Novel Method
}

\author{
Mustafa Inc ${ }^{1}$ and Ali Akgüll ${ }^{2,3}$ \\ ${ }^{1}$ Department of Mathematics, Science Faculty, Firat University, 23119 Elazı̆̆, Turkey \\ ${ }^{2}$ Department of Mathematics, Education Faculty, Dicle University, 21280 Diyarbakır, Turkey \\ ${ }^{3}$ Department of Mathematics, Texas A\&M University Kingsville, Kingsville, TX 78363, USA
}

Correspondence should be addressed to Ali Akgül; aliakgul00727@gmail.com

Received 25 November 2013; Revised 3 February 2014; Accepted 5 February 2014; Published 23 March 2014

Academic Editor: Hossein Jafari

Copyright (C) 2014 M. Inc and A. Akgül. This is an open access article distributed under the Creative Commons Attribution License, which permits unrestricted use, distribution, and reproduction in any medium, provided the original work is properly cited.

We demonstrate the efficiency of reproducing kernel Hilbert space method on the seventh-order boundary value problems satisfying boundary conditions. These results have been compared with the results that are obtained by variational iteration method (VIM), homotopy perturbation method (HPM), Adomian decomposition method (ADM), variation of parameters method (VPM), and homotopy analysis method (HAM). Obtained results show that our method is very effective.

\section{Introduction}

Consider the seventh-order boundary value problem [1-5]:

$$
u^{(7)}(x)=N(x, u(x)), \quad 0 \leq x \leq 1,
$$

with boundary conditions

$$
\begin{gathered}
u^{(i)}(0)=A_{i}, \quad i=0,1,2,3, \\
u^{(j)}(1)=B_{j}, \quad j=0,1,2 .
\end{gathered}
$$

The analytical solution of seventh-order differential equations are rarely exists in literature. However, there are various numerical methods for the solution of (1)-(2). The aim of this work is to apply reproducing kernel Hilbert space method (RKHSM) [6-28] to solve the seventh-order boundary value problems. Numerical results of the seventh-order boundary value problems have been obtained by this method in our work. This study shows that the proposed method can be considered as an alternative technique for solving linear and nonlinear problems in science and engineering [29-31].

The paper is organized as follows. Section 2 introduces several reproducing kernel spaces. We provide the main results and the exact and approximate solutions of (1)-(2) in Section 3. We have proved that the approximate solution converges to the exact solution uniformly. Some numerical experiments are illustrated in Section 4. There are some conclusions in the last section.

\section{Reproducing Kernel Spaces}

In this section, we define some useful reproducing kernel spaces.

Definition 1 . We define the space $H_{2}^{1}[0,1]$ by

$$
\begin{gathered}
H_{2}^{1}[0,1]=\{f \mid f \text { is absolutely continuous in }[0,1], \\
\left.f^{\prime}(x) \in L^{2}[0,1], x \in[0,1]\right\} .
\end{gathered}
$$

The inner product and the norm in $H_{2}^{1}[0,1]$ are defined, respectively, by

$$
\begin{gathered}
\langle f, g\rangle_{H_{2}^{1}}=f(0) g(0)+\int_{0}^{1} f^{\prime}(x) g^{\prime}(x) d x, \\
u, f \in H_{2}^{1}[0,1], \\
\|f\|_{H_{2}^{1}}=\sqrt{\langle f, f\rangle_{H_{2}^{1}}}, \quad f \in H_{2}^{1}[0,1] .
\end{gathered}
$$


The space $H_{2}^{1}[0,1]$ is a reproducing kernel space and its reproducing kernel function $r_{x}$ is given by

$$
r_{x}(y)= \begin{cases}1+x, & x \leq y \\ 1+y, & x>y\end{cases}
$$

Definition 2. We define the space $T_{2}^{8}[0,1]$ by the following:

$$
\begin{aligned}
T_{2}^{8}[0,1]=\{f \mid & f, f^{\prime}, f^{\prime \prime}, f^{(3)}, f^{(4)}, f^{(5)}, f^{(6)}, f^{(7)} \\
& \text { are absolutely continuous in }[0,1], \\
& f^{(8)} \in L^{2}[0,1], x \in[0,1], \\
& f(0)=f^{\prime}(0)=f^{\prime \prime}(0)=f^{(3)}(0)=f(1) \\
& \left.=f^{\prime}(1)=f^{\prime \prime}(1)=0\right\} .
\end{aligned}
$$

The inner product and the norm in $T_{2}^{8}[0,1]$ are defined, respectively, by

$$
\begin{aligned}
&\langle f, g\rangle_{T_{2}^{8}}= \sum_{i=0}^{7} f^{(i)}(0) g^{(i)}(0) \\
&+\int_{0}^{1} f^{(8)}(x) g^{(8)}(x) d x, \quad f, g \in T_{2}^{8}[0,1], \\
&\|f\|_{T_{2}^{8}}=\sqrt{\langle f, f\rangle_{T_{2}^{8}}}, \quad f \in T_{2}^{8}[0,1] .
\end{aligned}
$$

The space $T_{2}^{8}[0,1]$ is a reproducing kernel space; that is, for each fixed $y \in[0,1]$ and any $f \in T_{2}^{8}[0,1]$, there exists a function $R_{y}$ such that

$$
f=\left\langle f, R_{y}\right\rangle_{T_{2}^{8}}
$$

Theorem 3. The space $T_{2}^{8}[0,1]$ is a reproducing kernel Hilbert space whose reproducing kernel function $R_{y}$ is given by

$$
R_{y}(x)= \begin{cases}\sum_{i=1}^{16} c_{i}(y) x^{i-1}, & x \leq y, \\ \sum_{i=1}^{16} d_{i}(y) x^{i-1}, & x>y\end{cases}
$$

where $c_{i}(y)$ and $d_{i}(y)$ can be obtained by Maple 16 and proof of Theorem 3 is given in Appendix.

\section{Exact and Approximate Solutions of (1)-(2) in $T_{2}^{8}[0,1]$}

The solution of (1)-(2) is given in the reproducing kernel space $T_{2}^{8}[0,1]$. The linear operator

$$
L: T_{2}^{8}[0,1] \longrightarrow H_{2}^{1}[0,1]
$$

is bounded. After homogenizing the boundary conditions, we obtain

$$
\begin{gathered}
L v=M(x, v(x)), \quad 0 \leq x \leq 1, \\
v^{(i)}(0)=0, \quad i=0,1,2,3, \\
v^{(j)}(1)=0, \quad j=0,1,2 .
\end{gathered}
$$

We choose a countable dense subset $P=\left\{x_{i}\right\}_{i=1}^{\infty}$ in $[0,1]$ and let

$$
\Psi_{x}(y)=L^{*} r_{x}(y),
$$

where $L^{*}$ is conjugate operator of $L$ and $r_{x}$ is given by (5). Furthermore, for simplicity let $\Psi_{i}(x)=\Psi_{x_{i}}(x)$; namely,

$$
\Psi_{i}(x) \stackrel{\text { def }}{=} \Psi_{x_{i}}(x)=L^{*} r_{x_{i}}(x) .
$$

Now one can deduce the following lemmas.

Lemma 4. $\left\{\Psi_{i}(x)\right\}_{i=1}^{\infty}$ is complete system of $T_{2}^{8}[0,1]$.

Proof. For $f \in T_{2}^{8}[0,1]$, let $\left\langle f, \Psi_{i}\right\rangle=0(i=1,2, \ldots)$; that is,

$$
\left\langle f, L^{*} r_{x_{i}}\right\rangle=(L f)\left(x_{i}\right)=0 .
$$

Note that $\left\{x_{i}\right\}_{i=1}^{\infty}$ is the dense set in $[0,1]$; therefore, $(L f)(x)=$ 0 . It follows that $f(x)=0$ from the existence of $L^{-1}$.

Lemma 5. The following formula holds:

$$
\Psi_{i}(x)=\left(L_{\nu} R_{x}(\nu)\right)\left(x_{i}\right),
$$

where the subscript $v$ of operator $L_{v}$ indicates that the operator $L$ applies to function of $v$.

Proof. Consider the following:

$$
\begin{aligned}
\Psi_{i}(x) & =\left\langle\Psi_{i}(\xi), R_{x}(\xi)\right\rangle_{T_{2}^{8}}=\left\langle L^{*} r_{x_{i}}(\xi), R_{x}(\xi)\right\rangle_{T_{2}^{8}} \\
& =\left\langle\left(r_{x_{i}}\right)(\xi),\left(L_{\nu} R_{x}(\nu)\right)(\xi)\right\rangle_{H_{2}^{1}} \\
& =\left(L_{\nu} R_{x}(v)\right)\left(x_{i}\right) .
\end{aligned}
$$

This completes the proof.

Remark 6. The orthonormal system $\left\{\bar{\Psi}_{i}(x)\right\}_{i=1}^{\infty}$ of $T_{2}^{8}[0,1]$ can be derived from Gram-Schmidt orthogonalization process of $\left\{\Psi_{i}(x)\right\}_{i=1}^{\infty}$,

$$
\bar{\Psi}_{i}(x)=\sum_{k=1}^{i} \beta_{i k} \Psi_{k}(x), \quad\left(\beta_{i i}>0, i=1,2, \ldots\right),
$$

where $\beta_{i k}$ are orthogonal coefficients.

In the following, we will give the representation of the exact solution of (11) in the reproducing kernel space $T_{2}^{8}[0,1]$. 
Theorem 7. If $u$ is the exact solution of (11), then

$$
u=\sum_{i=1}^{\infty} \sum_{k=1}^{i} \beta_{i k} M\left(x_{k}, u\left(x_{k}\right)\right) \bar{\Psi}_{i}(x),
$$

where $\left\{x_{i}\right\}_{i=1}^{\infty}$ is a dense set in $[0,1]$.

Proof. From the (17) and uniqueness of solution of (11), we have

$$
\begin{aligned}
u & =\sum_{i=1}^{\infty}\left\langle u, \bar{\Psi}_{i}\right\rangle_{T_{2}^{8}} \bar{\Psi}_{i}=\sum_{i=1}^{\infty} \sum_{k=1}^{i} \beta_{i k}\left\langle u, L^{*} r_{x_{k}}\right\rangle_{T_{2}^{8}} \bar{\Psi}_{i} \\
& =\sum_{i=1}^{\infty} \sum_{k=1}^{i} \beta_{i k}\left\langle L u, r_{x_{k}}\right\rangle_{H_{2}^{1}} \bar{\Psi}_{i}=\sum_{i=1}^{\infty} \sum_{k=1}^{i} \beta_{i k}\left\langle M, r_{x_{k}}\right\rangle_{H_{2}^{1}} \bar{\Psi}_{i} \\
& =\sum_{i=1}^{\infty} \sum_{k=1}^{i} \beta_{i k} M\left(x_{k}, u\left(x_{k}\right)\right) \bar{\Psi}_{i}(x)
\end{aligned}
$$

This completes the proof.

Now the approximate solution $u_{n}$ can be obtained by truncating the $n$-term of the exact solution $u$ as

$$
u_{n}=\sum_{i=1}^{n} \sum_{k=1}^{i} \beta_{i k} M\left(x_{k}, u\left(x_{k}\right)\right) \bar{\Psi}_{i}(x) .
$$

Lemma 8. Assume $u$ is the solution of (11) and $r_{n}$ is the error between the approximate solution $u_{n}$ and the exact solution $u$. Then the error sequence $r_{n}$ is monotone decreasing in the sense of $\|\cdot\|_{T_{2}^{8}}$ and $\left\|r_{n}(x)\right\|_{T_{2}^{8}} \rightarrow 0$.

Proof. From (18) and (20), we obtain

$$
\left\|u-u_{n}\right\|_{T_{2}^{8}}=\left\|\sum_{i=n+1}^{\infty} \sum_{k=1}^{i} \beta_{i k} M\left(x_{k}, u\left(x_{k}\right)\right) \bar{\Psi}_{i}(x)\right\|_{T_{2}^{8}} .
$$

Thus

$$
\left\|u-u_{n}\right\|_{T_{2}^{8}} \longrightarrow 0, \quad n \longrightarrow \infty
$$

In addition

$$
\begin{aligned}
\left\|u-u_{n}\right\|_{T_{2}^{8}}^{2} & =\left\|\sum_{i=n+1}^{\infty} \sum_{k=1}^{i} \beta_{i k} M\left(x_{k}, u\left(x_{k}\right)\right) \bar{\Psi}_{i}(x)\right\|_{T_{2}^{8}}^{2} \\
& =\sum_{i=n+1}^{\infty}\left(\sum_{k=1}^{i} \beta_{i k} M\left(x_{k}, u\left(x_{k}\right)\right) \bar{\Psi}_{i}(x)_{T_{2}^{8}}\right)^{2} .
\end{aligned}
$$

Then, $\left\|u-u_{n}\right\|_{T_{2}^{8}}$ is monotonically decreasing in $n$.

Remark 9. The seventh-order boundary value problems have come out in construction engineering, beam column theory, and chemical reactions. Therefore solutions of the seventhorder boundary value problems are very important in the literature. The reproducing kernel function for seventh-order boundary value problem has not been calculated till now.

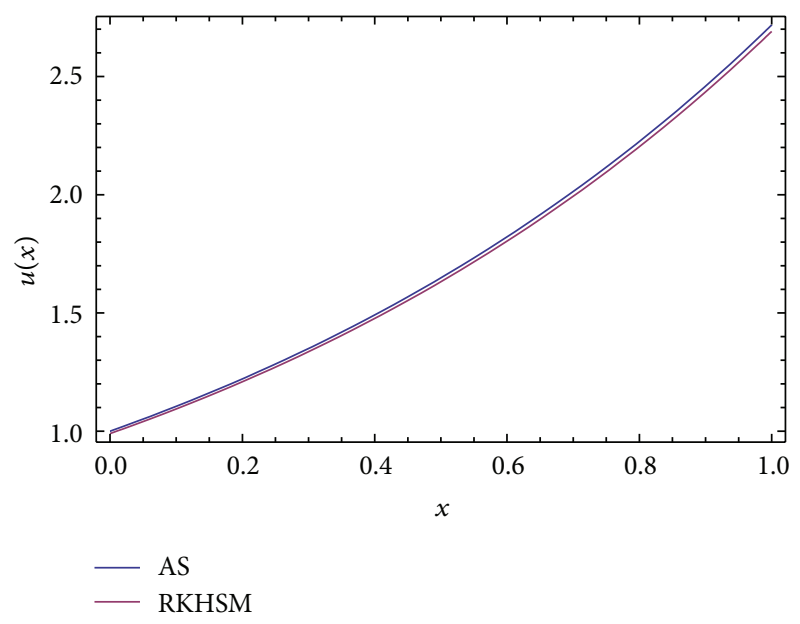

FIGURE 1: Comparison of analytical solution and RKHSM solution for Example 10

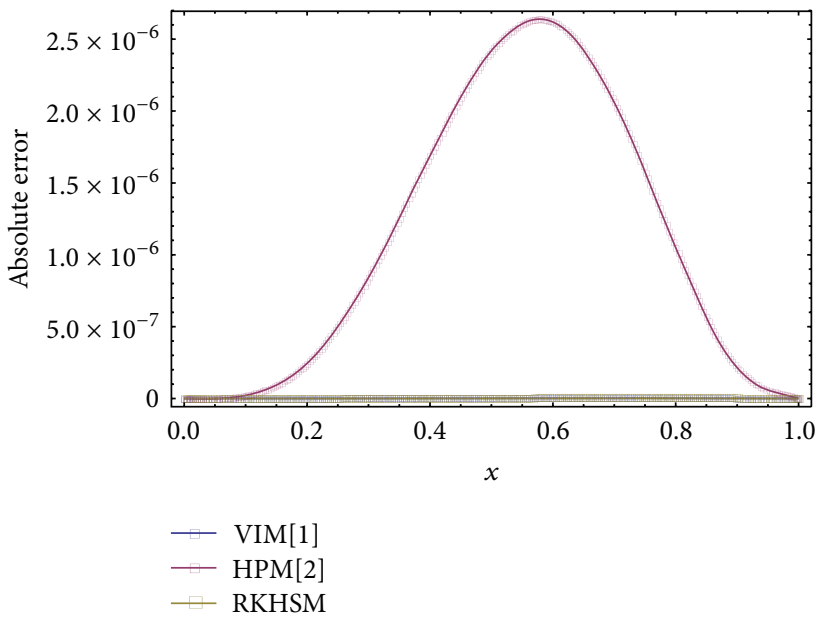

FIGURE 2: Comparison of absolute error of VIM, HPM, and RKHSM for Example 10.

All computations are performed by Maple 16. The RKHSM does not require discretization of the variables, that is, time and space, and it is not affected by computational round-off errors and one is not faced with necessity of large computer memory and time. The accuracy of the RKHSM for the seventh-order boundary value problems is controllable and absolute errors are small with present choice of $x$ (see Tables 1-6 and Figures 1-6). The obtained numerical results justify the advantage of this methodology. We gave transformations to homogenize the boundary conditions for all examples. Additionally, we improved our programme to find numerical results. As shown in Tables 1, 3, and 5 all the numerical results have been found in very short time.

\section{Numerical Results}

In this section, three numerical examples are provided to show the accuracy of the present method. 


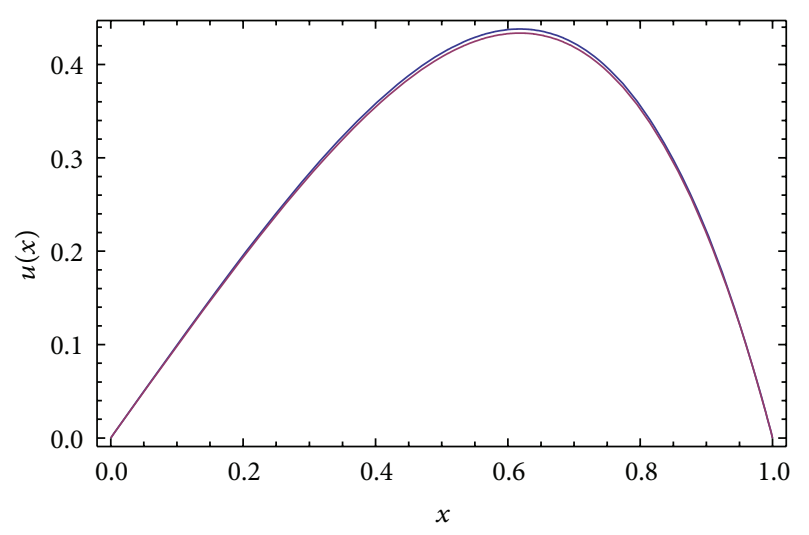

- AS

— RKHSM

FIGURE 3: Comparison of analytical solution and RKHSM solution for Example 11.

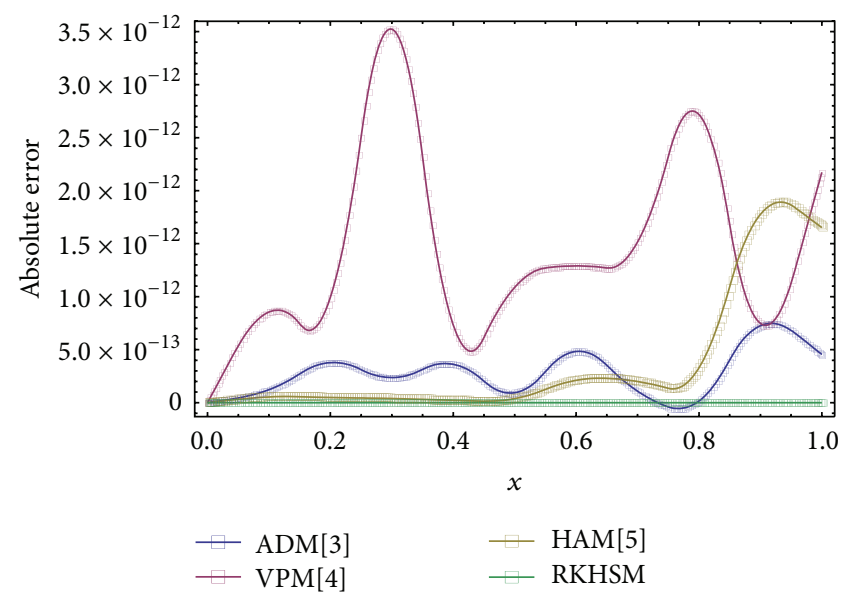

FIGURE 4: Comparison of absolute error of ADM, VPM, HAM, and RKHSM for Example 11.

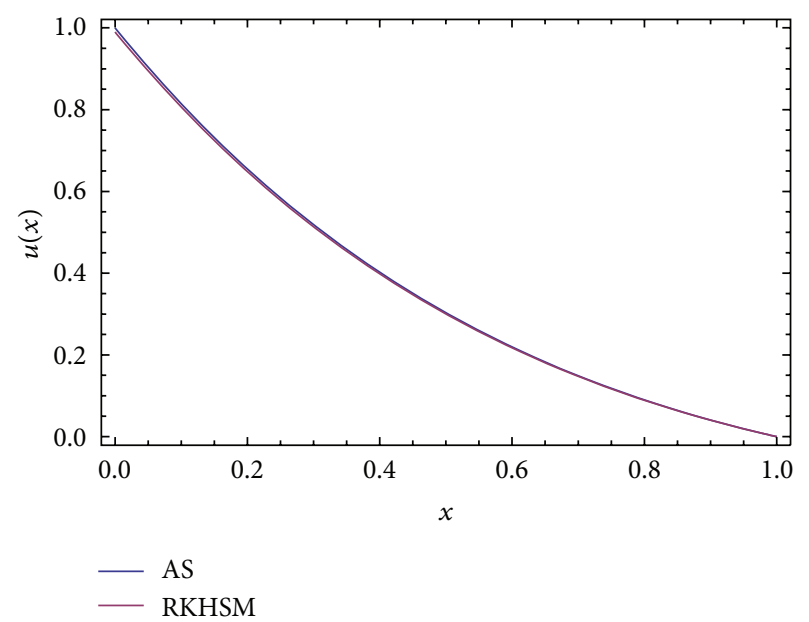

FIGURE 5: Comparison of analytical solution and RKHSM solution for Example 12.

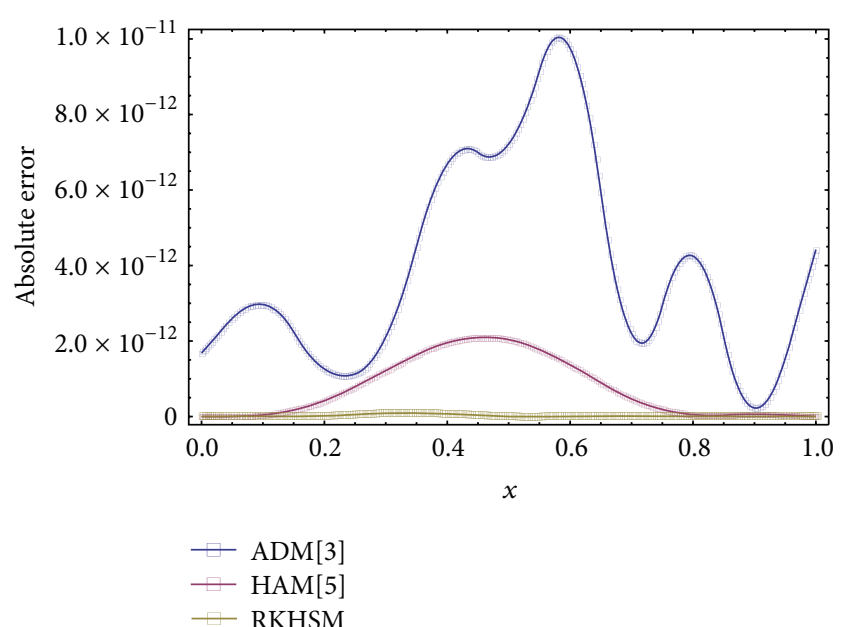

FIgURE 6: Comparison of absolute error of ADM, HAM, and RKHSM for Example 12.

Example 10. We first consider the seventh-order nonlinear boundary value problem:

$$
\begin{gathered}
u^{(7)}(x)=e^{-x} u^{2}(x), \quad 0<x<1, \\
u(0)=u^{\prime}(0)=u^{\prime \prime}(0)=u^{(3)}(0)=1, \\
u(1)=u^{\prime}(1)=u^{\prime \prime}(1)=e .
\end{gathered}
$$

The exact solution of (24) is given as [1]

$$
u(x)=e^{x} .
$$

After homogenizing the boundary conditions of (24), we obtain

$$
\begin{gathered}
v^{(7)}(x)=e^{-x}\left[v(x)+1+x+\frac{x^{2}}{2}+\frac{x^{3}}{6}+x^{4}\left(\frac{21}{2} e-\frac{57}{2}\right)\right. \\
\left.+x^{5}\left(\frac{87}{2}-16 e\right)+x^{6}\left(\frac{13}{2} e-\frac{53}{3}\right)\right]^{2}, \\
0 \leq x \leq 1, \\
v \begin{array}{c}
v(0)=v^{\prime}(0)=v^{\prime \prime}(0)=v^{(3)}(0)=0 \\
v(1)=v^{\prime}(1)=v^{\prime \prime}(1)=0,
\end{array}
\end{gathered}
$$

where we used the following transformation:

$$
\begin{aligned}
v(x)= & u(x)-1-x-\frac{x^{2}}{2}-\frac{x^{3}}{6}-x^{4}\left(\frac{21}{2} e-\frac{57}{2}\right) \\
& -x^{5}\left(\frac{87}{2}-16 e\right)-x^{6}\left(\frac{13}{2} e-\frac{53}{3}\right) .
\end{aligned}
$$

Using the RKHSM for this example we obtain Tables 1-2 and Figures 1-2. 
Example 11. We now consider the seventh-order linear BVP

$$
\begin{gathered}
u^{(7)}(x)=-u(x)-e^{x}\left(35+12 x+2 x^{2}\right), \quad 0 \leq x \leq 1, \\
u(0)=0, \quad u^{\prime}(0)=1, \\
u^{\prime \prime}(0)=0, \quad u^{(3)}(0)=-3, \\
u(1)=0, \quad u^{\prime}(1)=-e, \quad u^{\prime \prime}(1)=-4 e .
\end{gathered}
$$

The exact solution of (28) is given as [3]

$$
u(x)=x(1-x) e^{x}
$$

After homogenizing the boundary conditions of (28), we get

$$
\begin{aligned}
& v^{(7)}(x)+v(x)=-e^{x}\left(35+12 x+2 x^{2}\right)-x \\
&+\frac{x^{3}}{2}-x^{4}\left(3 e-\frac{17}{2}\right)-x^{5}\left(\frac{27}{2}-5 e\right) \\
&-x^{6}\left(2 e-\frac{11}{2}\right), \quad 0 \leq x \leq 1, \\
& v(0)= v^{\prime}(0)=v^{\prime \prime}(0)=v^{(3)}(0)=0, \\
& v(1)=v^{\prime}(1)=v^{\prime \prime}(1)=0,
\end{aligned}
$$

where, we used the following transformation

$$
\begin{aligned}
v(x)= & u(x)-x+x^{\frac{3}{2}}-x^{4}\left(3 e-\frac{17}{2}\right) \\
& -x^{5}\left(\frac{27}{2}-5 e\right)-x^{6}\left(2 e-\frac{11}{2}\right) .
\end{aligned}
$$

Using RKHSM for this example we obtain Tables 3-4 and Figures 3-4.

Example 12. Consider the following seventh-order nonlinear BVP

$$
\begin{array}{r}
u^{(7)}(x)=u(x) u^{\prime}(x)+e^{-2 x}\left(2+e^{x}(x-8)-3 x+x^{2}\right), \\
0 \leq x \leq 1,
\end{array}
$$

$$
\begin{gathered}
u(0)=1, \quad u^{\prime}(0)=-2, \\
u^{\prime \prime}(0)=3, \quad u^{(3)}(0)=-4, \\
u(1)=0, \quad u^{\prime}(1)=\frac{-1}{e}, \quad u^{\prime \prime}(1)=\frac{2}{e} .
\end{gathered}
$$

The exact solution of (32) is given as [3]

$$
u(x)=(1-x) e^{-x}
$$

After homogenizing the boundary conditions of (32), we have

$$
\begin{gathered}
v^{(7)}(x)-\left(-2+3 x-2 x^{2}+4 x^{3}\left(\frac{6}{e}-2\right)+5 x^{4}\left(4-\frac{11}{e}\right)+6 x^{5}\left(\frac{5}{e}-\frac{11}{6}\right)\right) v(x) \\
-\left(1-2 x+\frac{3}{2} x^{2}-\frac{2}{3} x^{3}+x^{4}\left(\frac{6}{e}-2\right)+x^{5}\left(4-\frac{11}{e}\right)+x^{6}\left(\frac{5}{e}-\frac{11}{6}\right)\right) v^{\prime}(x) \\
=\left[\begin{array}{c}
\left.\left(1-2 x+\frac{3}{2} x^{2}-\frac{2}{3} x^{3}+x^{4}\left(\frac{6}{e}-2\right)+x^{5}\left(4-\frac{11}{e}\right)+x^{6}\left(\frac{5}{e}-\frac{11}{6}\right)\right)\right] \\
\left(-2+3 x-2 x^{2}+4 x^{3}\left(\frac{6}{e}-2\right)+5 x^{4}\left(4-\frac{11}{e}\right)+6 x^{5}\left(\frac{5}{e}-\frac{11}{6}\right)\right)
\end{array}\right] \\
+v(x) v^{\prime}(x)+e^{-2 x}\left(2+e^{x}(x-8)-3 x+x^{2}\right), \quad 0 \leq x \leq 1, \\
v(0)=v^{\prime}(0)=v^{\prime \prime}(0)=v^{(3)}(0)=0, \\
v(1)=v^{\prime}(1)=v^{\prime \prime}(1)=0,
\end{gathered}
$$

where we used the following transformation:

$$
\begin{aligned}
u(x)= & v(x)+1-2 x+\frac{3}{2} x^{2}-\frac{2}{3} x^{3} \\
& +x^{4}\left(\frac{6}{e}-2\right)+x^{5}\left(4-\frac{11}{e}\right)+x^{6}\left(\frac{5}{e}-\frac{11}{6}\right) .
\end{aligned}
$$

Using RKHSM for this example we obtain Tables 5-6 and Figures 1-6.

Remark 13. Using our method we chose 36 points on $[0,1]$. In Tables 1-6, we computed the absolute errors $\left|u(x, t)-u_{n}(x, t)\right|$ at the points $\left\{\left(x_{i}\right): x_{i}=i, i=0.0,0.1, \ldots, 1.0\right\}$. The RKHSM tested on three problems, one linear and two nonlinear. A comparison with VIM [1], HPM [2], ADM [3], VPM [4], and 
TABLE 1: Numerical results for Example 10 (time (s): 1.645).

\begin{tabular}{lcccc}
\hline$x$ & Exact solution & Approximate solution & Absolute error & Relative error \\
\hline 0.0 & 1.0 & 1.0 & 0.0 & 0.0 \\
0.1 & 1.1051709180756476248 & 1.1051709180727538232 & $2.893 \times 10^{-12}$ & $2.618 \times 10^{-12}$ \\
0.2 & 1.2214027581601698339 & 1.2214027581330885422 & $2.708 \times 10^{-11}$ & $2.217 \times 10^{-11}$ \\
0.3 & 1.3498588075760031040 & 1.3498588075089984856 & $6.700 \times 10^{-11}$ & $4.963 \times 10^{-11}$ \\
0.4 & 1.4918246976412703178 & 1.4918246975765762858 & $6.469 \times 10^{-11}$ & $4.336 \times 10^{-11}$ \\
0.5 & 1.6487212707001281468 & 1.6487212707474912552 & $4.736 \times 10^{-11}$ & $2.872 \times 10^{-11}$ \\
0.6 & 1.8221188003905089749 & 1.8221188006600701498 & $2.695 \times 10^{-10}$ & $1.479 \times 10^{-10}$ \\
0.7 & 2.0137527074704765216 & 2.0137527079512967073 & $4.808 \times 10^{-10}$ & $2.387 \times 10^{-10}$ \\
0.8 & 2.2255409284924676046 & 2.2255409289689961077 & $4.765 \times 10^{-10}$ & $2.141 \times 10^{-10}$ \\
0.9 & 2.4596031111569496638 & 2.4596031113394646558 & $1.825 \times 10^{-10}$ & 0.0 \\
1.0 & 2.7182818284590452354 & 2.7182818284590452354 & & $7.420 \times 10^{-11}$ \\
\hline
\end{tabular}

TABLE 2: Comparison of absolute error of HPM, VIM, and RKHSM for Example 10.

\begin{tabular}{lccc}
\hline$x$ & Absolute error [2] & Absolute error [1] & Absolute error [RKHSM] \\
\hline 0.0 & 0.0 & 0.0 & 0.0 \\
0.1 & $2.15 \times 10^{-8}$ & $3.8478 \times 10^{-12}$ & $2.8938016 \times 10^{-12}$ \\
0.2 & $2.45 \times 10^{-7}$ & $1.2366 \times 10^{-10}$ & $2.70812917 \times 10^{-11}$ \\
0.3 & $8.42 \times 10^{-7}$ & $2.7788 \times 10^{-10}$ & $6.70046184 \times 10^{-11}$ \\
0.4 & $1.69 \times 10^{-6}$ & $7.5864 \times 10^{-10}$ & $6.46940320 \times 10^{-11}$ \\
0.5 & $2.42 \times 10^{-6}$ & $1.1571 \times 10^{-9}$ & $4.73631084 \times 10^{-11}$ \\
0.6 & $2.62 \times 10^{-6}$ & $1.3132 \times 10^{-9}$ & $2.695611749 \times 10^{-10}$ \\
0.7 & $2.06 \times 10^{-6}$ & $1.2228 \times 10^{-9}$ & $4.808201857 \times 10^{-10}$ \\
0.8 & $1.05 \times 10^{-6}$ & $6.6023 \times 10^{-10}$ & $4.765285031 \times 10^{-10}$ \\
0.9 & $2.14 \times 10^{-7}$ & $1.6534 \times 10^{-10}$ & $1.825149920 \times 10^{-10}$ \\
1.0 & $4.44 \times 10^{-16}$ & $1.3265 \times 10^{-11}$ & 0.0 \\
\hline
\end{tabular}

HAM [5] was made and it was seen that the present method yields good results (see Tables 1-6 and Figures 1-6).

\section{Conclusion}

In this paper, we introduced an algorithm for solving the seventh-order problem with boundary conditions. For illustration purposes, we chose three examples which were selected to show the computational accuracy. It may be concluded that the RKHSM is very powerful and efficient in finding exact solution for a wide class of boundary value problems. The approximate solution obtained by the present method is uniformly convergent. Clearly, the series solution methodology can be applied to much more complicated nonlinear differential equations and boundary value problems. However, if the problem becomes nonlinear, then the RKHSM does not require discretization or perturbation and it does not make closure approximation. Results of numerical examples show that the present method is an accurate and reliable analytical method for the seventh-order boundary value problem.

\section{Appendix}

Proof of Theorem 3. Let $f \in T_{2}^{8}[0,1]$. By Definition 2 we have

$$
\left\langle f, R_{y}\right\rangle_{T_{2}^{8}}=\sum_{i=0}^{7} f^{(i)}(0) R_{y}^{(i)}(0)+\int_{0}^{1} f^{(8)}(x) R_{y}^{(8)}(x) d x .
$$

Through several integrations by parts for (A.1) we have

$$
\begin{aligned}
\left\langle f, R_{y}\right\rangle_{T_{2}^{8}}= & \sum_{i=0}^{7} u^{(i)}(0)\left[R_{y}^{(i)}(0)-(-1)^{(7-i)} R_{y}^{(15-i)}(0)\right] \\
& +\sum_{i=0}^{7}(-1)^{(7-i)} u^{(i)}(1) R_{y}^{(15-i)}(1) \\
& +\int_{0}^{1} u(x) R_{y}^{(16)}(x) d x .
\end{aligned}
$$

Note that property of the reproducing kernel

$$
\left\langle u, R_{y}\right\rangle_{T_{2}^{8}}=u(y) \text {. }
$$


TABLE 3: Numerical results for Example 11 (time (s): 3.123).

\begin{tabular}{lccc}
\hline$x$ & Exact solution & Approximate solution & Absolute error \\
\hline 0.0 & 0.0 & 0.0 & 0.0 \\
0.1 & 0.099465382626808286232 & 0.099465382626808285898 & $3.34 E-19$ \\
0.2 & 0.19542444130562717342 & 0.19542444130562716451 & $8.91 E-18$ \\
0.3 & 0.28347034959096065184 & 0.28347034959096048526 & $1.6658 E-16$ \\
0.4 & 0.35803792743390487627 & 0.35803792743390483786 & $3.841 E-17$ \\
0.5 & 0.41218031767503203670 & 0.41218031767503200826 & $2.844 E-17$ \\
0.6 & 0.43730851209372215398 & 0.43730851209372205668 & $9.730 E-17$ \\
0.7 & 0.42288806856880006954 & 0.42288806856879998460 & $8.494 E-17$ \\
0.8 & 0.35608654855879481674 & 0.35608654855879479280 & $2.394 E-17$ \\
0.9 & 0.22136428000412546974 & 0.22136428000412540564 & $6.410 E-17$ \\
1.0 & 0.0 & $1.24053 E-20$ & $1.24053 E-20$ \\
\hline
\end{tabular}

TABLE 4: Comparison of absolute error of VPM, ADM, HAM, and RKHSM.

\begin{tabular}{lcccc}
\hline$x$ & Absolute error [4] & Absolute error [3] & Absolute error [5] & Absolute error [RKHSM] \\
\hline 0.0 & 0.0 & 0.0 & 0.0 & 0.0 \\
0.1 & $8.55607 E-13$ & $1.23082 E-13$ & $5.39291 E-14$ & $3.34 E-19$ \\
0.2 & $9.94041 E-12$ & $3.7792 E-13$ & $4.85167 E-14$ & $8.91 E-18$ \\
0.3 & $3.52244 E-11$ & $2.37421 E-13$ & $3.92464 E-14$ & $1.6658 E-16$ \\
0.4 & $7.3224 E-10$ & $3.62099 E-13$ & $2.21489 E-14$ & $3.841 E-17$ \\
0.5 & $1.08769 E-10$ & $9.39249 E-14$ & $3.84137 E-14$ & $2.844 E-17$ \\
0.6 & $1.29035 E-10$ & $4.82947 E-13$ & $2.10831 E-13$ & $9.730 E-17$ \\
0.7 & $1.51466 E-10$ & $1.09135 E-13$ & $1.99785 E-13$ & $8.494 E-17$ \\
0.8 & $2.717974 E-10$ & $1.64868 E-14$ & $3.29736 E-13$ & $2.394 E-17$ \\
0.9 & $7.48179 E-10$ & $7.25975 E-13$ & $1.77622 E-12$ & $6.410 E-17$ \\
1.0 & $2.1729 E-09$ & $4.54747 E-13$ & $1.65159 E-12$ & $1.24053 E-20$ \\
\hline
\end{tabular}

Now, if

$$
\begin{gathered}
R_{y}^{(4)}(0)+R_{y}^{(11)}(0)=0 \\
R_{y}^{(5)}(0)-R_{y}^{(10)}(0)=0 \\
R_{y}^{(6)}(0)+R_{y}^{(9)}(0)=0 \\
R_{y}^{(7)}(0)-R_{y}^{(8)}(0)=0 \\
R_{y}^{(8)}(1)=0 \\
R_{y}^{(9)}(1)=0 \\
R_{y}^{(10)}(1)=0 \\
R_{y}^{(11)}(1)=0 \\
R_{y}^{(12)}(1)=0
\end{gathered}
$$

then (A.2) implies that

$$
R_{y}^{(16)}(x)=\delta(x-y)
$$

when $x \neq y$, then

$$
R_{y}^{(16)}(x)=0
$$

and therefore

$$
R_{y}(x)= \begin{cases}\sum_{i=1}^{16} c_{i}(y) x^{i-1}, & x \leq y, \\ \sum_{i=1}^{16} d_{i}(y) x^{i-1}, & x>y .\end{cases}
$$

Since

$$
R_{y}^{(16)}(x)=\delta(x-y)
$$

we have

$$
\begin{gathered}
R_{y^{+}}^{(k)}(y)=R_{y^{-}}^{(k)}(y), \quad k=0, \ldots, 14, \\
R_{y^{+}}^{(15)}(y)-R_{y^{-}}^{(15)}(y)=1 .
\end{gathered}
$$

Since $R_{y} \in T_{2}^{8}[0,1]$, it follows that

$$
\begin{aligned}
R_{y}(0) & =R_{y}^{\prime}(0)=R_{y}^{\prime \prime}(0)=R_{y}^{(3)}(0)=R_{y}(1) \\
& =R_{y}^{\prime}(1)=R_{y}^{\prime \prime}(1)=0 .
\end{aligned}
$$

From (A.4)-(A.10), the unknown coefficients $c_{i}(y)$ and $d_{i}(y)(i=1,2, \ldots, 16)$ can be obtained. This completes the proof. 
TABLE 5: Numerical results for Example 12 (time (s): 5.234).

\begin{tabular}{lccc}
\hline$x$ & Exact solution & Approximate solution & Absolute error \\
\hline 0.0 & 1.0 & 1.0 & 0.0 \\
0.1 & 0.81435367623236361584 & 0.81435367623236697064 & $3.35480 \times 10^{-15}$ \\
0.2 & 0.65498460246238548694 & 0.65498460246237032242 & $1.516452 \times 10^{-14}$ \\
0.3 & 0.51857275447720250625 & 0.51857275447711724998 & $8.525627 \times 10^{-14}$ \\
0.4 & 0.40219202762138358044 & 0.40219202762145521926 & $7.163882 \times 10^{-14}$ \\
0.5 & 0.30326532985631671180 & 0.30326532985631655134 & $1.6046 \times 10^{-16}$ \\
0.6 & 0.21952465443761057305 & 0.21952465443760682026 & $3.75279 \times 10^{-15}$ \\
0.7 & 0.14897559113742285441 & 0.14897559113743255406 & $9.69965 \times 10^{-15}$ \\
0.8 & 0.089865792823444318286 & 0.089865792823449635588 & $5.317302 \times 10^{-15}$ \\
0.9 & 0.040656965974059911188 & 0.040656965974059021714 & $8.89474 \times 10^{-16}$ \\
1.0 & 0.0 & $2.708848 \times 10^{-22}$ & $2.708848 \times 10^{-22}$ \\
\hline
\end{tabular}

TABLE 6: Comparison of absolute error of ADM, HAM, and RKHSM.

\begin{tabular}{lccc}
\hline$x$ & Absolute error [3] & Absolute error [5] & Absolute error [RKHSM] \\
\hline 0.0 & $1.67932 E-12$ & 0.0 & 0.0 \\
0.1 & $2.96696 E-12$ & $4.15223 E-14$ & $3.3548 E-15$ \\
0.2 & $1.26055 E-12$ & $4.18332 E-13$ & $1.516452 E-14$ \\
0.3 & $2.10898 E-12$ & $1.21736 E-12$ & $8.525627 E-14$ \\
0.4 & $6.68926 E-12$ & $1.95471 E-12$ & $7.163882 E-14$ \\
0.5 & $7.21923 E-12$ & $2.03731 E-12$ & $1.6046 E-16$ \\
0.6 & $9.75339 E-12$ & $1.37063 E-12$ & $3.75279 E-15$ \\
0.7 & $2.19552 E-12$ & $4.66988 E-13$ & $9.69965 E-15$ \\
0.8 & $4.24917 E-12$ & $4.8378 E-14$ & $5.317302 E-15$ \\
0.9 & $2.27311 E-13$ & $6.00561 E-14$ & $8.89474 E-16$ \\
1.0 & $4.42298 E-12$ & $1.29172 E-15$ & $2.708848 E-22$ \\
\hline
\end{tabular}

\section{Disclosure}

This paper is a part of the Ph.D. thesis of Ali Akgül.

\section{Conflict of Interests}

The authors declare that they do not have any competing or conflict of interests.

\section{References}

[1] S. S. Siddiqi, G. Akram, and M. Iftikhar, "Solution of seventh order boundary value problems by variational iteration technique," Applied Mathematical Sciences, vol. 6, no. 94, pp. 46634672, 2012.

[2] F. Mabood and M. Idrees, "Homotopy perturbation method and seventh-order boundary value problems," International Journal of Applied Mathematical Research, vol. 2, no. 1, pp. 70-75, 2013.

[3] S.S. Siddiqi and M. Iftikhar, "Solution of seventhorder boundary value problems using Adomian decomposition method," http://arxiv.org/abs/1301.3603.

[4] S. S. Siddiqi and M. Iftikhar, "Solutions of seventh-order boundary value problems by variation of parameters method," Research Journal of Applied Sciences, Engineering and Technology, vol. 5, no. 1, pp. 176-179, 2013.
[5] S. S. Siddiqi and M. Iftikhar, "Numerical solution of higher order boundary value problems," Abstract and Applied Analysis, vol. 2013, Article ID 427521, 12 pages, 2013.

[6] M. Cui and Y. Lin, Nonlinear Numerical Analysis in the Reproducing Kernel Space, Nova Science, New York, NY, USA, 2009.

[7] H. Yao and M. Cui, "A new algorithm for a class of singular boundary value problems," Applied Mathematics and Computation, vol. 186, no. 2, pp. 1183-1191, 2007.

[8] W. Wang, M. Cui, and B. Han, "A new method for solving a class of singular two-point boundary value problems," Applied Mathematics and Computation, vol. 206, no. 2, pp. 721-727, 2008.

[9] Y. Zhou, Y. Lin, and M. Cui, "An efficient computational method for second order boundary value problems of nonlinear differential equations," Applied Mathematics and Computation, vol. 194, no. 2, pp. 354-365, 2007.

[10] X. Lü and M. Cui, "Analytic solutions to a class of nonlinear infinite-delay-differential equations," Journal of Mathematical Analysis and Applications, vol. 343, no. 2, pp. 724-732, 2008.

[11] Y.-l. Wang and L. Chao, "Using reproducing Kernel for solving a class of partial differential equation with variable-coefficients," Applied Mathematics and Mechanics, vol. 29, no. 1, pp. 129-137, 2008.

[12] F. Li and M. Cui, "A best approximation for the solution of onedimensional variable-coefficient Burgers' equation," Numerical 
Methods for Partial Differential Equations, vol. 25, no. 6, pp. 1353-1365, 2009.

[13] S. Zhou and M. Cui, "Approximate solution for a variablecoefficient semilinear heat equation with nonlocal boundary conditions," International Journal of Computer Mathematics, vol. 86, no. 12, pp. 2248-2258, 2009.

[14] X. Lv and M. Cui, "An efficient computational method for linear fifth-order two-point boundary value problems," Journal of Computational and Applied Mathematics, vol. 234, no. 5, pp. 1551-1558, 2010.

[15] W. Jiang and M. Cui, "Constructive proof for existence of nonlinear two-point boundary value problems," Applied Mathematics and Computation, vol. 215, no. 5, pp. 1937-1948, 2009.

[16] J. Du and M. Cui, "Constructive proof of existence for a class of fourth-order nonlinear BVPs," Computers \& Mathematics with Applications, vol. 59, no. 2, pp. 903-911, 2010.

[17] M. Cui and H. Du, "Representation of exact solution for the nonlinear Volterra-Fredholm integral equations," Applied Mathematics and Computation, vol. 182, no. 2, pp. 1795-1802, 2006.

[18] Y. Lin and M. Cui, "A numerical solution to nonlinear multipoint boundary value problems in the reproducing Kernel space," Mathematical Methods in the Applied Sciences, vol. 34, no. 1, pp. 44-47, 2011.

[19] M. Mohammadi and R. Mokhtari, "Solving the generalized regularized long wave equation on the basis of a reproducing Kernel space," Journal of Computational and Applied Mathematics, vol. 235, no. 14, pp. 4003-4014, 2011.

[20] B. Y. Wu and X. Y. Li, "A new algorithm for a class of linear nonlocal boundary value problems based on the reproducing Kernel method," Applied Mathematics Letters, vol. 24, no. 2, pp. 156-159, 2011.

[21] M. Inc and A. Akgül, "The reproducing Kernel Hilbert space method for solving Troesch's problem," Journal of the Association of Arab Universities for Basic and Applied Sciences, vol. 14, no. 1, pp. 19-27, 2013.

[22] M. Inc, A. Akgül, and F. Geng, "Reproducing Kernel Hilbert space method for solving Bratu's problem," Bulletin of the Malaysian Mathematical Sciences Society. In press.

[23] M. Inc, A. Akgül, and A. Kiliçman, "Explicit solution of telegraph equation based on reproducing Kernel method," Journal of Function Spaces and Applications, vol. 2012, Article ID 984682, 23 pages, 2012.

[24] M. Inc, A. Akgül, and A. Kılıçman, "A new application of the reproducing Kernel Hilbert space method to solve MHD Jeffery-Hamel flows problem in nonparallel walls," Abstract and Applied Analysis, vol. 2013, Article ID 239454, 12 pages, 2013.

[25] M. Inc, A. Akgül, and A. Kiliçman, "A novel method for solving $\mathrm{KdV}$ equation based on reproducing Kernel Hilbert space method," Abstract and Applied Analysis, vol. 2013, Article ID 578942, 11 pages, 2013.

[26] M. Inc, A. Akgül, and A. Kılıçman, "Numerical solutions of the second-order one-dimensional telegraph equation based on reproducing Kernel Hilbert space method," Abstract and Applied Analysis, vol. 2013, Article ID 768963, 13 pages, 2013.

[27] G. Akram and H. U. Rehman, "Numerical solution of eighth order boundary value problems in reproducing Kernel space," Numerical Algorithms, vol. 62, no. 3, pp. 527-540, 2013.
[28] W. Wang, B. Han, and M. Yamamoto, "Inverse heat problem of determining time-dependent source parameter in reproducing Kernel space," Nonlinear Analysis: Real World Applications, vol. 14 , no. 1, pp. 875-887, 2013.

[29] Y. Khan and F. Austin, "Application of the laplace decomposition method to nonlinear homogeneous and non-homogenous advection equations," Zeitschrift für Naturforschung A, vol. 65, no. 10, pp. 849-853, 2010.

[30] Y. Khan and Q. Wu, "Homotopy perturbation transform method for nonlinear equations using He's polynomials," Computers \& Mathematics with Applications, vol. 61, no. 8, pp. 19631967, 2011.

[31] Y. Khan, "An effective modification of the laplace decomposition method for nonlinear equations," International Journal of Nonlinear Sciences and Numerical Simulation, vol. 10, no. 11-12, pp. 1373-1376, 2009. 


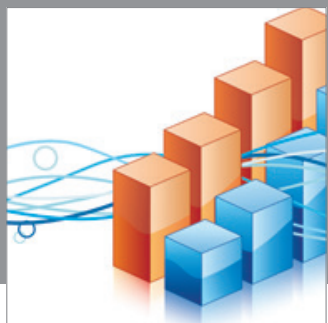

Advances in

Operations Research

mansans

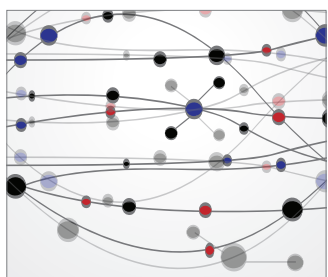

The Scientific World Journal
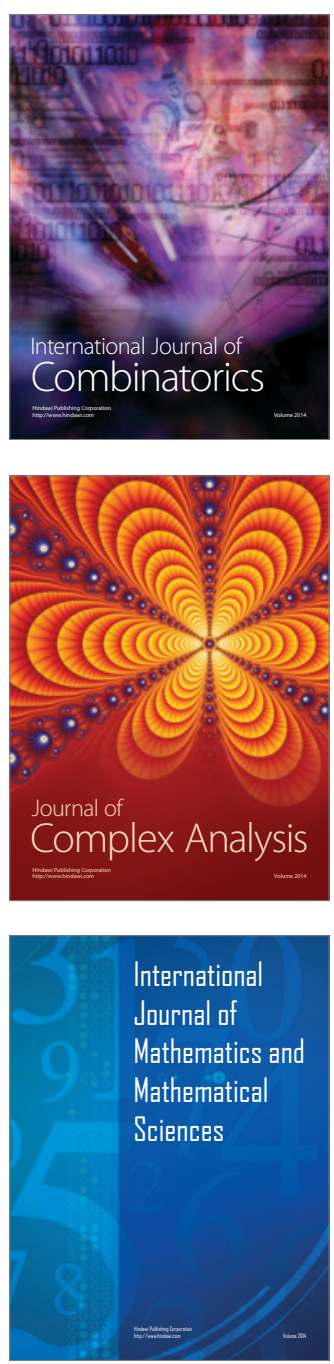
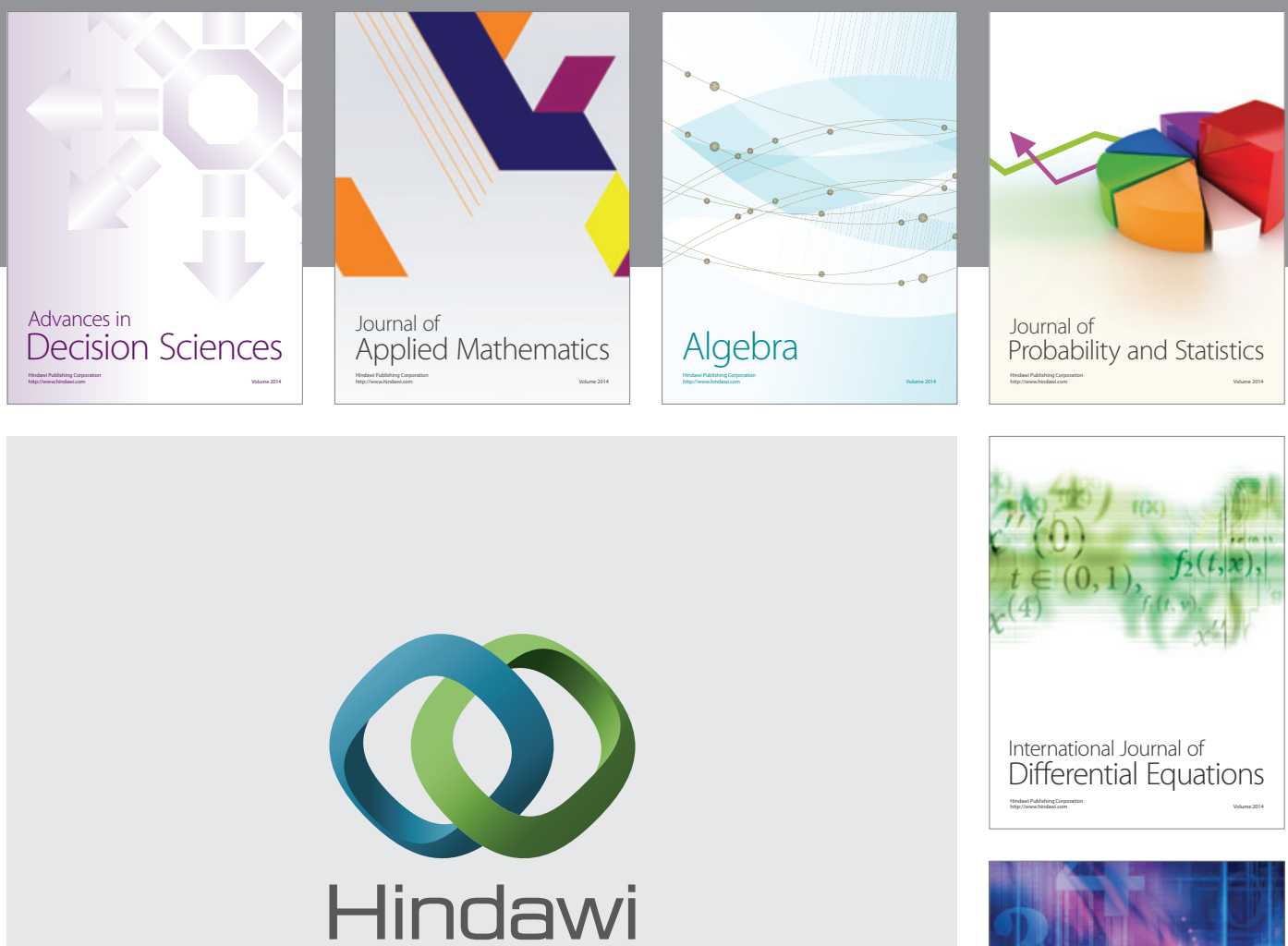

Submit your manuscripts at http://www.hindawi.com
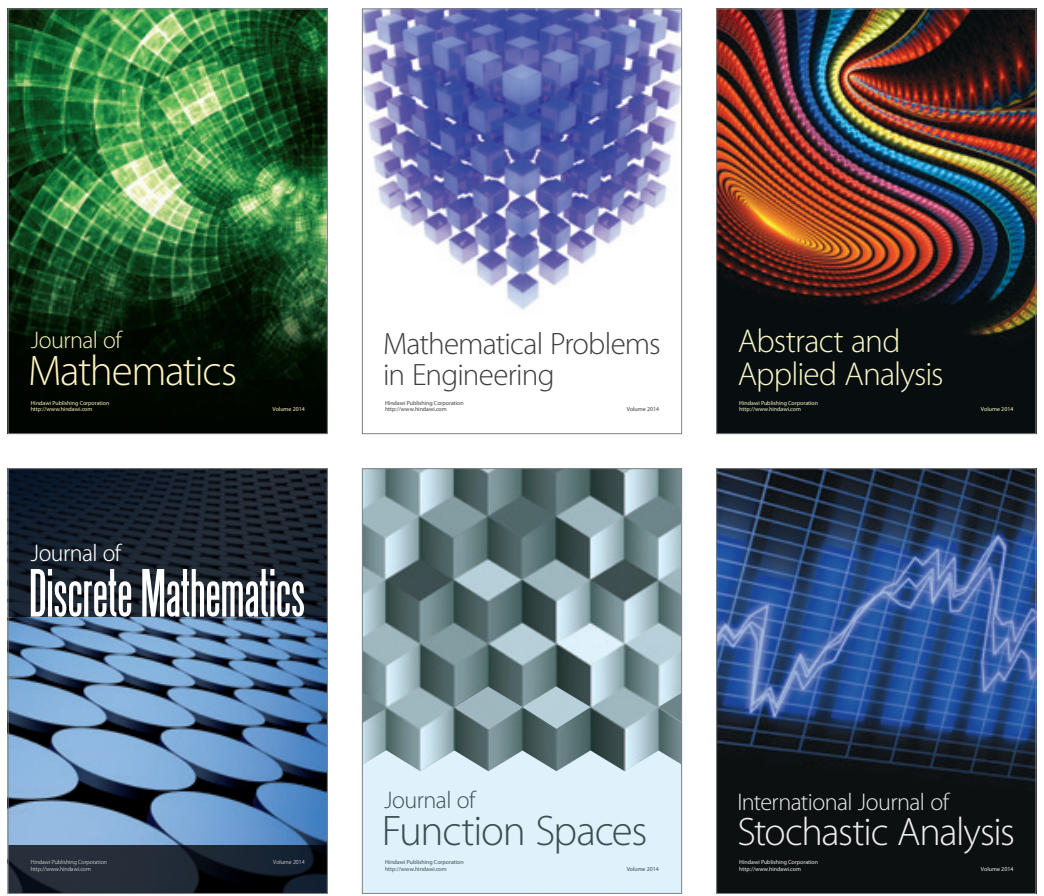

Journal of

Function Spaces

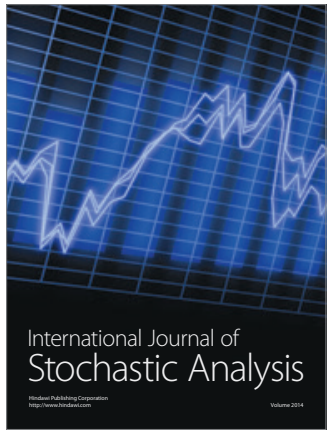

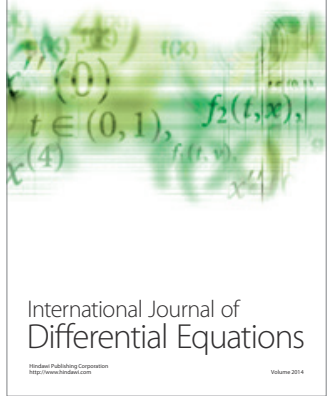
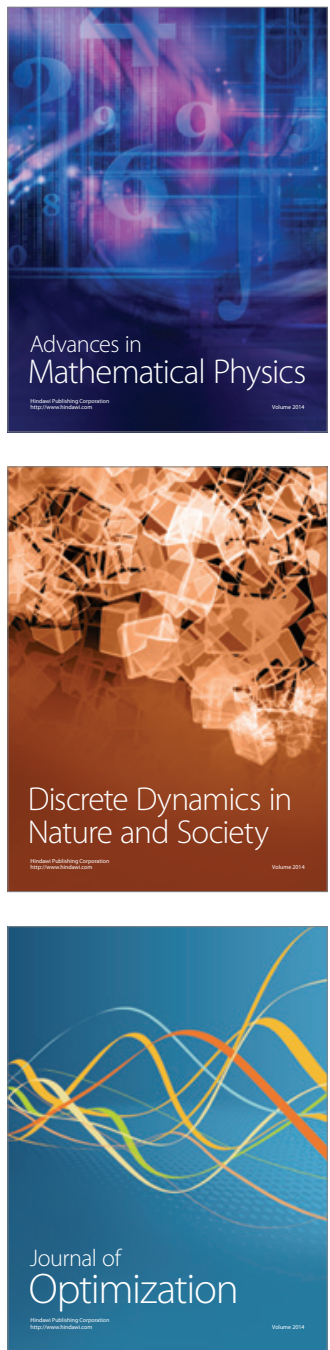